\title{
Collaborative and Robot-Based Plug \& Produce for Rapid Reconfiguration of Modular Production Systems
}

\author{
Michael Wojtynek ${ }^{1}$, Hendrik Oestreich ${ }^{1}$, Oliver Beyer ${ }^{2}$, Sebastian Wrede ${ }^{1}$
}

\begin{abstract}
The manufacturing of individualized products down to batch size 1 poses ongoing challenges for the design and integration of future production systems. Today's production lines with a high degree of automation achieve high efficiency, but usually come with high costs for adaptation to product variants. In order to combine full automation with high flexibility, we propose a concept for the dynamic composition of automation components in a modular production system that facilitates the rapid adaptation of collaborative and robotsupported manufacturing processes. To achieve this, we integrate self-descriptive automation components at runtime into the control architecture of the production system using a Plugand-Produce approach. While the location and orientation of automation components in the modular production system are derived from physical human-robot interaction, the adaptation and verification of the robot behavior is made possible through a simulation-based planning subsystem. Once this dynamic reconfiguration process by the machine setter is finished, the adapted production process is executed in a fully automated way with high efficiency. A case study carried out in an industrial collaboration project on flexible assembly demonstrates the benefits of the presented approach.
\end{abstract}

\section{INTRODUCTION}

Current developments in industry show a radical change in production and automation. More and more digitalization takes place in manufacturing machinery, production systems, equipment and fabrication processes. The accompanying process shows a shift from large scale to a small batch production, which is slowly replacing mass production with individual and customized products. Reasons for a small batch production can be found in short lifecycles of products, which have to establish themselves on fluctuating and unpredictable markets. The high customization rate combined with special desires of the customer significantly increase production costs and challenge the traditional manufacturing methods.

In order to tackle these upcoming challenges, we present a modular production system, which aims to achieve a high process flexibility facilitating a collaborative robot comprising the cognitive strength and professional experience of factory workers. Further, we combine the principles of a fully automated production system with an interactive and individually configurable production process.

The flexibility of the modular production system is enabled by a unified treatment of automation components that try to meet the requirements of a versatile production process.

\footnotetext{
${ }^{1}$ Research Institute for Cognition and Robotics (CoR-Lab), Bielefeld University, Universitätsstr. 25, 33615 Bielefeld, Germany mwojtynek, hoestreich, swrede@cor-lab. uni-bielefeld.de

${ }^{2}$ HARTING IT Software Development, Marienwerderstr. 2, 32339 Espelkamp, Germany Oliver.Beyer@harting.com
}

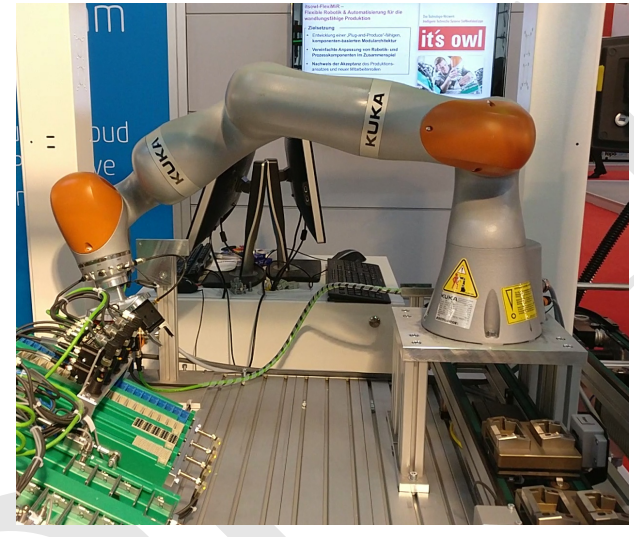

Fig. 1: Modular production system with a collaborative robot during an assembly task on the Hannover Industry Fair in 2017.

In particular, any equipment used for manufacturing in the modular production system, is predefined and described in terms of software interfaces and hardware structure. We call this representation of soft- and hardware aspects $\mathrm{Ab}$ stract Automation Component (AAC) in the following. The key concept behind AACs is the dynamic composition of components at runtime with a subsequent parametrization. The process of adding AACs at runtime is called Plug-andProduce and constitutes the prerequisite for our approach, in which components perform a manual or automated selfconfiguration.

For versatile production of individualized, complex and high quality goods, we consider the initial configuration of the modular production system and the reconfiguration of an existing process the most important and frequent operations in contemporary industrial scenarios. Collaborative robots are often used to compensate the demands of versatile productions. However, once a robot is part of the mentioned production process, the integration of newly added AACs has a potential impact on the production procedure and behavior of the system.

It is mandatory to adapt the robot motion and behavior rapidly to the changed process to keep integration time and costs as well as programming efforts as low as possible. The use of collaborative lightweight robots increases enormously the variability in a production process and enables with six or seven degrees of freedom an excellent reachability of objects in the workspace, where humans and robots work side-byside in various collaborative scenarios (e.g. sequenced task, sharing workspace). Due to the lightweight design and based on safety logic and functions, collaborative robots can be considered as safe with regards to collisions and harms 
towards the human worker or the factory environment.

In our approach the shop floor worker is highly involved in the reconfiguration process and carries out the setup of the modular production cell, the AACs and the robot behavior. During this reconfiguration, the worker receives assistance in form of information from a software wizard shown on a user control panel to perform the reconfiguration in the proper way and sequence. This guidance lowers the comprehension barrier of the technical system, especially if a complex machine like a robot is part of it or if AACs demand special purpose parametrization.

Therefore, we introduce a novel approach for a seamless integration capability of a collaborative robot into a coherent Plug-and-Produce system that solves the above problems using the following methods: (i) modularization of hard- and software components, (ii) unification of software interfaces and hardware structure, (iii) self-description of automation components to specify functionalities and (iv) a simulation based approach for calculating collision free robot paths in changing environment combined with a stepwise real world motion execution.

In the following we present the related work in Section II. Section III describes a case study we performed with an industry-oriented production process for an assembly of industrial connectors. Afterwards, we describe in Section IV the procedure of changing an existing production process reconfiguring and extending by a new AAC such as a camera for quality inspection. Section $\mathrm{V}$ presents the architecture of our system. Finally, consequences and an outlook can be found in Section VI we conclude in Section VII.

\section{RELATED WORK}

Several publications report on research targeting flexible manufacturing in the context of modular production cells, reconfiguration of soft- and hardware components and utilization of robots for rapid production of customized products in constrained workspaces.

Scheifele [1] presents a memory concept for modular production systems with exchangeable process modules, which contain their configuration and provide a module-description and their I/O capabilities. Friedrich [2] shows a decentralized approach for a standardized description of automation modules. The control system is able to identify the modules and execute a self-configuration procedure. Further, a guideline for implementing Industry 4.0 [3], formulates the goal of a component-driven modular production, to put a digitalized production into practice.

The Plug-and-Produce concept is considered as a fundamental requirement for automatic configuration and rearranging modern production systems [4] and has been a widely investigated topic in research: Pfrommer et al. [5], [6] analyze how the definition and formalization of skills can support the reconfiguration of manufacturing systems. Furthermore, the use of a robot in an assembly process entails new challenges: The integration of new automation components has an enormous impact on the robot. Existing motions in the production process would lead to collisions and additional

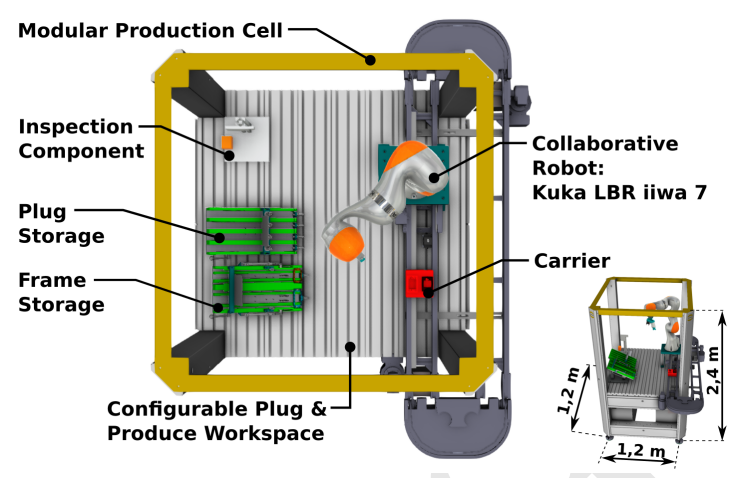

Fig. 2: Top view and layout of a modular production cell for Plugand-Produce purposes using a collaborative robot.

movements have to be planned. The adaption of the robot behavior in the process can either be derived automatically or with the support of the shop floor worker. Therefore, different approaches have been researched, including programming by demonstration [7] or even more generic robot skill modelling [8], [9], [10].

To integrate automation components into an existing process, they have to provide some information about their capabilities, interfaces and dimensions. Keddis proposes in [11] to use standardized models that are not only applied during design but also during runtime. This concept was investigated in the context of capability modelling of PLCs (Programmable Logic Controller) while claiming that it can be generalized to robot control programs [12].

Schleipen et al. [13] state that besides the models for the components also models for the product itself and the production process would be necessary for a successful Plug-and-Produce realization. They recommend the usage of AutomationML for the description of products, resources and processes.

Moreover, Zoitl et al. chose the interesting approach of minimizing the programming effort by generating the machine programs from a production plan [14]. This is alike to our method where we modularize and encapsulate functionality to make it attachable to production plans directly. Also closely related to our approach is the work of Goto et al. [15] who used mealy finite state machines to model a collaborative human-robot assembly process, including error handling and branched control structures. Other research takes a different approach through modelling a Plug-andProduce framework based on multi-agents [16].

Wrede et al. [17] present a service orchestration for modular production systems with the use of standardized business process models. The presented approach aims at the integration of a modular production line from shop floor to business level. Similarly Horbach [18] and Jentsch [19] focus on a higher level planning concept. In contrast, this current work shifts the focus from vertical integration and higher level planing to flexible reconfiguration and fast commissioning of one production cell using a collaborative robot and the skills of the operator. 


\section{CASE Study}

In the following section, we first describe the basic production process for an assembly of industrial connectors as part of our case study. Afterwards we present the procedure, how an existing production process can be changed, reconfigured and extended by a new AAC such as a camera for quality inspection. The challenge hereby is to link and extend the existing production plan with the provided functions of the new AAC. More challenges arise during integration of AACs that are related to the robot behavior and motion: robot motions have to be adjusted concerning path planning, collision avoidance and task completion. The basic production process and application scenario consist the following parts (shown in Fig. 2):

- Modular production cell, which defines the workspace, provides mounting points, contains the machine periphery (e.g. power, fieldbus, compressed air) and safety control as well as sensors for product identification

- Collaborative lightweight robot such as the Kuka LBR IIWA 7 with two-finger grippers for grasping objects like hinged frames and plugs

- Storages for keeping and providing process material

- Carrier that runs on a conveyor belt to transport assembled products out of the production cell

- Control Panel for interactions with the operator and displaying instructions

The mentioned connectors, assembled here as an exemplary use case, consist of a hinged metal frame and mostly plastic insets. These insets cover a wide product range that aims for application-specific tasks in industry and meet individual customer needs. For instance, there are insets for power, network connection or compressed air. Besides the basic, robot-based and automated production process the case study investigates the reconfiguration and extension of a present process. The reconfiguration is a conducted experiment that examines the technical opportunities for rapid adaption to requirements of a flexible production. Section IV describes the reconfiguration process, whilst the the basic production process is explained in the following.

At the beginning of the process, an empty charge carrier coded with an RFID tag (Radio-Frequency Identification) is moved on the conveyor belt into the production cell and the required production process is started based on the RFID tag. The hinged frames as well as the plugs of the connectors are provided in storages, which can be reached and picked by the collaborative robot. Therefore, the robot has a tandem end-effector with one gripper for each picking task. Robot positions and poses to perform the production task are defined in advance. Fig. 3 demonstrates in detail the assembly steps. According to the production plan the robot starts by picking the hinged frame and places it on the carrier. Afterwards force-based robot control is needed to open up the hinged frame, which was stocked in closed state inside the storage. Force-based control means that the current robot motion is finished when a certain force-threshold specific to the current process step is reached. As soon as the hinged

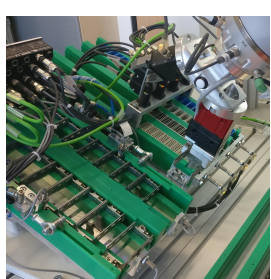

(a)

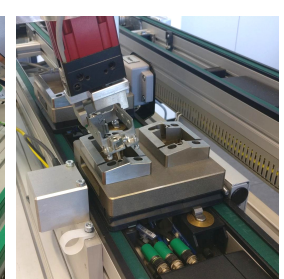

(b)

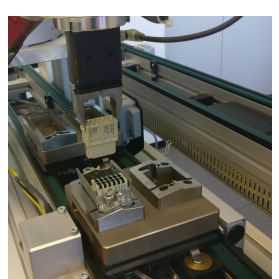

(c)
Fig. 3: Basic production process of the case study: (a) robot picks hinged frame from storage, (b) placement on carrier, (c) force-based assembly of insets in hinged frame.

frame has been prepared, the robot picks the insets one by one and places them into the hinged frame. The type and sequence of the plugs are encoded in the individual customer order represented in the production process model. Once the lightweight robot has placed all plugs in the frame, the hinged frame is closed with further force-based robot motions and the assembly is finished. Finally, the conveyor belt transports the carrier with the assembled product out of the cell and a new order can be processed.

In the following, an extension of the production process serves as the reference example to illustrate our reconfiguration approach: the dynamic integration of a new AAC and the adaption of the robot behavior based on our Plug-andProduce concept.

\section{INTERACTIVE RECONFIGURATION}

In this section we show a simple and effective way to reconfigure an existing production process utilizing a collaborative robot. Key ideas of the Plug-and-Produce concept are:

1) Modularization of automation components used in the workspace of the modular production cell. Measuring points on components enable the computation of the position inside workspace.

2) Dynamic integration of AACs, which means that automation components can be integrated or exchanged at runtime.

3) A collaborative robot and cognitive capabilities of the operator are used for exploration of the environment and localization of AACs.

4) Simulation and path planning support the integration of AACs regarding robot motions.

5) Functional knowledge provided about services, communication nodes and geometrical details of the AAC. These structured datasets are called self-description.

6) A unified connector enables a wired connection and a self-identification towards the production system.

The reconfiguration workflow is described in the BPMN2.0 modelling language with standardised syntax and semantics that facilitates process automation. The executable model of the Plug-and-Produce process is visualized in Fig. 4, which organizes the process in several subprocesses and lanes, which show a strong interaction between each other. Lanes represent the processing of a specific task, such as operator assignments or simulation services. The AAC, an 


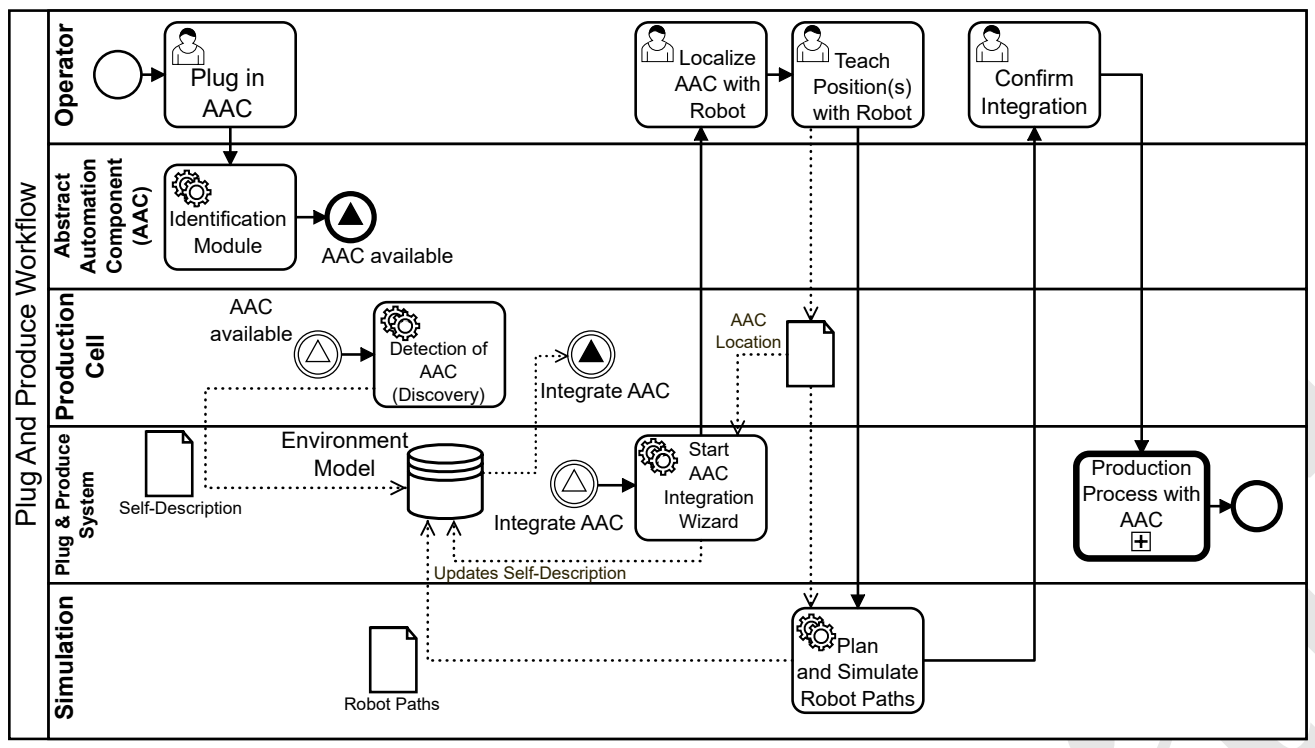

Fig. 4: Dynamic Plug-and-Produce workflow for interactive reconfiguration of an abstract automation component.

inspection camera, that is integrated and reconfigured during runtime is shown in Fig. 5. It is as an exemplary AAC in order to ensure quality aspects. The reconfiguration task consists of locating the AAC and adjusting robot motions and paths. Adjusting robot paths is necessary since the product must be moved in front of the camera lens to determine by image processing whether the hinged frame is closed properly.

At the beginning of the reconfiguration process the operator places the AAC inside the workspace and connects it with the unified connector to the modular production cell. This process is part of the Plug-and-Produce concept and is represented by a user task (human icon inside the process activity) in the reconfiguration workflow. Once the AAC is connected to the production cell, it indicates its presence automatically (gearbox icon) to the system and unlocks a Plug-and-Produce wizard on the control panel of the modular production cell. During the whole Plug-andProduce process the operator is guided and assisted with helpful instructions by the wizard on the control panel. From now on, the modular production system is ready for an interactive AAC integration and the parametrization can be started.

The next assistance step of the wizard asks the operator to provide essential geometrical details about the environment and locate the AAC using the collaborative robot. Therefore the robot serves as a flexible, collaborative and precise measuring device. The benefits of this method are that neither manual measurement by the operator is needed nor external sensors for automated localization of the AAC must be integrated.

For the exploration of the workspace the operator is instructed to insert a special purpose tool for localization into the gripper of the robot. The collaborative robot is switched then to a gravity compensation mode, where the operator moves the robot and its end-effector freely inside the workspace. Gravity compensation means that the weight of the robot and its tool is compensated and the user can guide the robot by applying forces on the end-effector or the robot joints.

The task of the operator is to move the localization tool towards the calibration points of the AAC (compare Fig. 5) that are indicated on the control screen. Storing the calibration by using the kinematic chain of the robot allows to calculate the position and orientation of the AAC inside the workspace of the modular production cell.

An integral part of the Plug-and-Produce concept is to utilize a 3D-model-based robot and environment simulation for visualizing the reconfiguration state and for generating collision free paths of robot motions. The geometrical 3Dmodel of the AAC as well as the position are automatically integrated and updated inside the $3 \mathrm{D}$ representation after the exploration procedure with the collaborative robot. The operator benefits from a clear visual representation of the internal state in the modular production cell represented as 3D model and part of the integration wizard. This method of localization and visual representation enables keeping the discrepancy between the real world and 3D simulated workspace to a minimum as possible.

The successful localization of the AAC allows an automated path planning calculation. Furthermore, the simulation shows on the control panel the calculated path to the AAC inside the production process and asks the operator for confirmation. Based on the visual impression of the 3D simulation the operator decides whether to accept the calculated robot motion, which was suggested by the path planning algorithm. In case the robot motions appear ineffective or unsafe, the operator can request a new path. The computed paths are collision free in simulation, otherwise the path computation fails and the AAC has to be repositioned and relocated. The operator proofs the absence of collisions in the real world by executing each robot motion step by step with reduced velocity and applied impedance mode (compliant spring-damper behavior). These increase safety drastically 


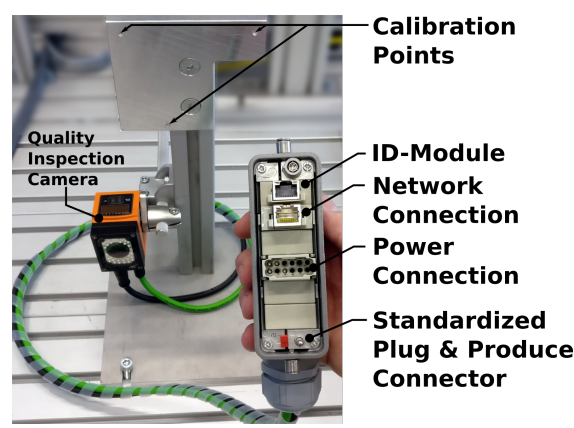

Fig. 5: Automated Plug-and-Produce component with a unified hardware connector.

and prevent damage with the industrial environment of the modular production cell. Once these steps have been finished, the operator can deploy and start the production process in working speed with the newly integrated AAC.

\section{System ARchitecture}

This section presents the system architecture facilitating the interactive reconfiguration workflow outlined in Sec. IV. The service-oriented software design supports loose coupling, re-usability and dynamic composition of components. In the following, the minimal set of core services required for fully automated production are introduced first. Subsequently, the additional services are explained, which are necessary for Plug-and-Produce of AACs at runtime.

\section{A. Core Services}

The core service components for an automated and robotbased production are presented in an internal block diagram in SysML notation shown in Fig. 6. The process engine, the collaborative robot and the storage are the core hard- and software components within the system. The main flow items are customers orders, which are received by the modular production system, and after internal processing, an assembled product is delivered as outcome.

The process engine represents the essential core service within the overall system architecture. It receives outstanding assembly orders and is responsible for the interpretation as well as execution of production plans. These production plans are represented in the system as BPMN2.0 process models. The automated interpretation and execution of these production process models is achieved in a software component that extends the open-source Camunda process engine.

Different types of robot motions, coordinates and joint configurations can be defined directly as specific BPMN activities with corresponding parameters in the production process models. The robot plugin, running in the process engine, integrates the robot state to the running process instance and sets up communication ports as needed by the robot activities. For example, a directed output port (port type: Task Frame) connects the process engine with the service component of the robot controller if task frame data needs to be exchanged as part of a motion command. Each task frame describes an end-effector position and orientation

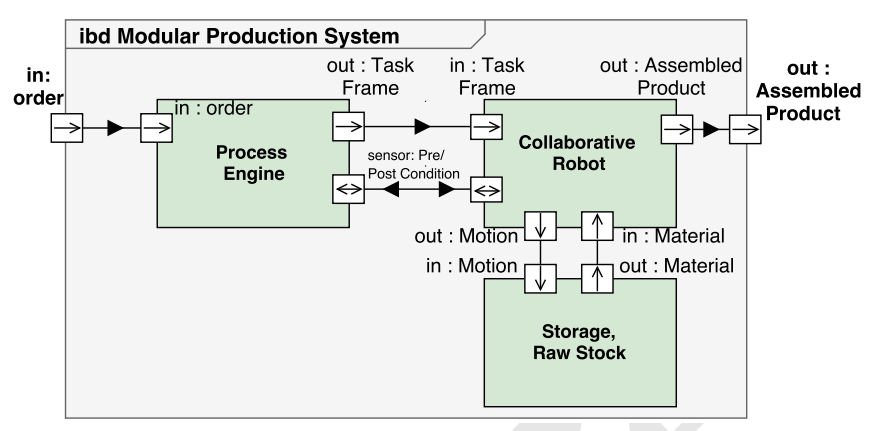

Fig. 6: System architecture overview for an automated high-level production process with core services.

for the robot such that the robot controller can calculate a motion between a start and goal frame.

The necessary task frames are programmed interactively using kinesthetic teaching before a new production plan is executed. The robot plugin checks that each robot activity is bound to existing task frames and suspends execution if a requested task frame is undefined at runtime. Further, bidirectional pre- and postconditions have to be satisfied by the robot to accomplish an activity in the production plan. An exemplary precondition is the checking for security approval of a robot motion. A post condition checks if an activity in the process instance, such as a robot movement to a desired goal position has been executed successfully and whether the process can be continued or if an error handling strategy needs to be performed. If every task frame can be resolved and the conditions are positively evaluated, the process engine is able to execute the modeled robot activities in the production process without further human intervention resembling a fully automated manufacturing process.

The internal block diagram of our core system architecture as shown in Fig. 6 highlights that the robot motions modeled in the production plans and executed by the process engine eventually lead to a manipulation of material. Here, the production process models typically exhibit robot-based pick and place activities for the assembly of insets and hinged frames that are provided by the storage. A successful manipulation of material results in an assembled product and is shown as the outcome of the internal block diagram.

\section{B. Plug-and-Produce Services}

To enable a dynamic Plug-and-Produce of AACs at runtime, the presented high-level system architecture is extended by services of an ID-Module, a Discovery Service, an environment model and a simulation of the robot and its surrounding (see Fig. 7).

Concerning the Plug-and-Produce hardware, each AAC is equipped with a unified connector that enables the wired connection with the modular production cell, which is shown in Fig. 5. Main parts of the AAC connector are plugs for power transmission and wired networking as well as the Identification Module (ID-Module) containing the selfdescription of the hardware component. The ID-Module contains an ARM-based embedded system, which entails a runtime container for the Plug-and-Produce software services of 


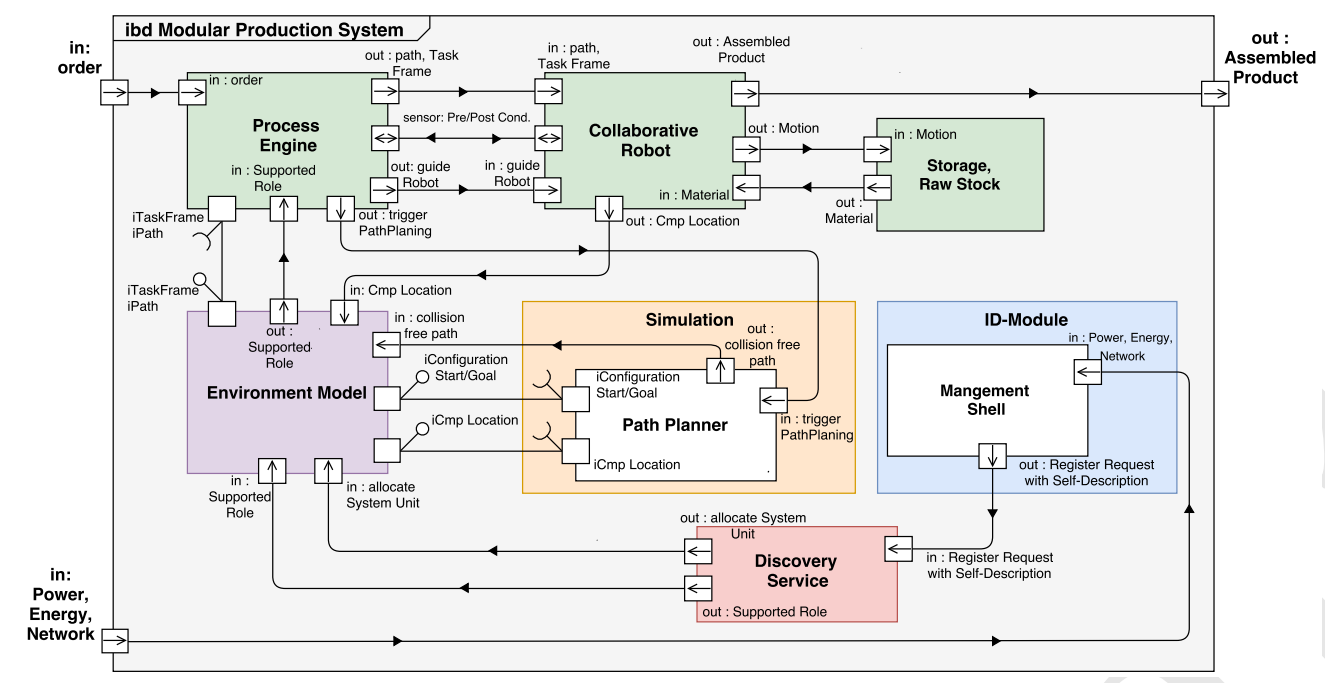

Fig. 7: System architecture of an automated production with Plug-and-Produce services used at runtime.

the Management Shell. To perform registration requests from the ID-Module, Multicast-DNS (mDNS) is used to resolve IP communication endpoints. The Management Shell provides a service interface that registers the AAC availability to the Discovery Service. This interface is modeled as a set of REST-based services using the Web Application Description Language (WADL).

Registration requests from the ID-Module are received by the Discovery Service, which records the availability of a specific component. Once an AAC has been registered to the Discovery Service, its self-description is parsed in order to utilize its service functionalities during production. Besides its WADL description, the Automation Markup Language (AML) is used to represent the component structure, its functional role as well as required communication with other components and its 3D-model information. The AAC itself is described here as an abstract System Unit Class in AML terminology and instantiated as a concrete hardware device. Moreover, the Supported Role class describes its services for task completion, which can be integrated and executed by the process engine.

The environment model manages the instantiated System Unit and the Supported Roles of an AAC using a graph database (Neo4j). Further, it maintains the representation of the current environment state in the modular production cell with available Plug-and-Produce components and their services. The current state of the environment model is observable by other services following a Publisher-Subscriber pattern. For instance, a message event is generated and sent to the process engine, when a System Unit and its Supported Role is allocated.

Accordingly, the environment model plugin for the process engine is designed to receive events from the underlying graph database at runtime, so that through the start and intermediate signals defined in the BPMN2-based process models, the resulting system behavior is dynamically selected. For instance, in the presented system, the environment model triggers a defined, reusable and guided assistance process for interactive reconfiguration of an AAC. The different steps and details of the assistance routine are encoded in this specific process model that is executed by the process engine upon the received signal or message event. Furthermore, the process engine is able to switch the robot state at certain activities of the assistance process into a collaborative mode such as the gravity compensating. The required network communication with a loose coupling between the participants, such as the communication between the process engine and environment model, was realized using the Robotics Service Bus (RSB) [20], which implements a middleware communication with publish-subscribe and event-based patterns.

A path planner for the robot motion behavior is provided by the simulation block, which may be also triggered by the process engine. An interface to the environment model supplies the path planner with the robots' start and goal configuration for a certain motion activity in the process plan (port type: iConfiguration). In addition, the environment model feeds the path planner with locations (position and orientation) of the newly integrated AACs inside the workspace (port type: iComp Location). Based on this data, the path planner computes collision free motion paths for the robot and stores these inside the representation and configuration of the environment model. For assembling a product, the process engine executes activities from the production plan using the stored paths from the environment model for performing collision free robot motions.

The interplay of the presented services yield an architecture supporting the requirements of a Plug-and-Produce and reconfiguration process. The core services of the automated production were extended with elements that address identification and integration of AACs into the modular production system to perform localization and dynamic path planning using a collaborative robot, human input and an environment representation combined with a simulation.

\section{Consequences AND Outlook}

In order to validate and demonstrate the presented Plugand-Produce concepts with its interactive reconfiguration 
workflow we developed a system prototype that was exhibited at the Hannover Industrial fair in 2017. The resulting prototype is depicted in Fig. 1 and a video of the full reconfiguration process is available at [21].

The realization and implementation of the Plug-andProduce concept has shown valuable results for the reconfiguration of an existing robot-based production process. The presented approach separates the functional view from the technical level. This means that complex robot operations and programming efforts are encapsulated from the operator. The encapsulation concept allows the operator to focus on the task completion without considering process parameters like robot coordinates or integration of automation modules. However, it has to be mentioned that current process plans during reconfiguration lacks flexibility. At the present state the operator has to perform the collaboration with the robot as presented in the assistance plan. Changes of the production plan or automated validity checks can not be performed by the operator on the current development state and have to investigated by future work.

Although this contribution has examined qualitative and architectural aspects of the modular production system, future research will introduce metrics for quantitative measurements of the human operator using a collaborative robot. Quantitative performance indicators can be registered by recording and evaluating values like the completion rate of an industrial task. Task time, error tracing and improvements of the Plugand-Produce system based on click-events will lead to further scientific findings towards human-robot-collaboration.

\section{CONCLUSION}

Within this contribution we motivated an extended Plugand-Produce approach for the flexible manufacturing of individualized products with the goal to reduce the costs for adaptation to product variants. Typically, the integration of new automation components in robot-based production cells is challenging and the adaptation of the required robot behavior is time-consuming. Therefore, we introduced an extended system architecture that copes with this problem by modularization and abstraction of hard- and software into dynamically composable components, so called Abstract Automation Components. This approach allows to integrate the self-descriptive abstract automation components at runtime into the control architecture of the production system. While the location and orientation of physical instances of automation components are derived from human-robot interaction, the adaptation and verification of the robot behavior is made possible through a simulation-based planning subsystem. Once this dynamic reconfiguration process that is typically executed by the machine setter is finished, the adapted production process may be executed again in a fully automated way with high efficiency. Self-description of modules, utilization of human-robot interaction, a workspace simulation and flexible but standard-based process models are combined in an efficient methodology, which addresses successfully the problem of changing processes and environments in individualized production.

\section{ACKNOWLEDGMENT}

This work received funding from the German Federal Ministry of Education and Research (BMBF) within the Leading-Edge Cluster "Intelligent Technical Systems OstWestfalenLippe" (it's OWL) managed by the Project Management Agency Karlsruhe (PTKA), and from "Digital in NRW - Das Kompetenzzentrum für den Mittelstand”.

\section{REFERENCES}

[1] S. Scheifele, J. Friedrich, A. Lechler, and A. Verl, "Flexible, Selfconfiguring Control System for a Modular Production System," Procedia Technology, vol. 15, pp. 398-405, 2014.

[2] J. Friedrich, S. Scheifele, A. Verl, and A. Lechler, "Flexible and Modular Control and Manufacturing System," in Procedia CIRP, vol. 33. Elsevier B.V., 2015, pp. 115-120.

[3] VDMA Insustrie 4.0 Forum, "Guideline Industrie 4.0: Guiding principles for the implementation of Industrie 4.0 in small and medium sized businesses," Plattform Industrie 4.0, 2016.

[4] ABB, "'Plug and produce' business systems with Industrial IT," 2001.

[5] J. Pfrommer et al., "Modelling and orchestration of service-based manufacturing systems via skills," in 19th IEEE International Conference on Emerging Technologies and Factory Automation, ETFA 2014, 2014.

[6] J. Pfrommer, D. Stogl et al., "Plug \& produce by modelling skills and service-oriented orchestration of reconfigurable manufacturing systems," At-Automatisierungstechnik, vol. 63, no. 10, pp. 790-800, 2015.

[7] S. Wrede, C. Emmerich, R. Grünberg, A. Nordmann, A. Swadzba, and J. Steil, "A User Study on Kinesthetic Teaching of Redundant Robots in Task and Configuration Space," Journal of Human-Robot Interaction, vol. 2, no. 1, pp. 56-81, 2013.

[8] M. Naumann, K. Wegener, and R. D. Schraft, "Control architecture for robot cells to enable Plug'n'Produce," in Proceedings - IEEE International Conference on Robotics and Automation, Roma, Italy, 2007, pp. 287-292.

[9] S. Bøgh, O. S. Nielsen, M. R. Pedersen, V. Krüger, and O. Madsen, "Aalborg Universitet Does your Robot have Skills?" in 43rd International Symposium on Robotics. Taipei, Taiwan: VDE Verlag GMBH, 2012 , p. 6.

[10] M. R. Pedersen, Robot Skills for Transformable Manufacturing Systems, 2014, no. November.

[11] N. Keddis et al., "Towards adaptable manufacturing systems," in Proceedings of the IEEE International Conference on Industrial Technology, 2013.

[12] Kainz et al., "Control Programs 4.0 Structure and Format of Control Programs in the Context of," in AUTOMATION, 2014.

[13] M. Schleipen et al., "Requirements and concept for Plug-and-Work," at Automatisierungstechnik, pp. 801-820, 2015.

[14] A. Zoitl, G. Kainz, and N. Keddis, "Production Plan-Driven Flexible Assembly Automation Architecture," in International Conference on Industrial Applications of Holonic and Multi-Agent Systems, 2013, pp. 49-58.

[15] H. Goto, J. Miura, and J. Sugiyama, "Human-Robot Collaborative Assembly by On-line Human Action Recognition Based on an FSM Task Model," in International Conference on Human-Robot Interaction Workshop on Collaborative Manipulation, 2013, pp. 1-6.

[16] M. Onori, N. Lohse, J. Barata, and C. Hanisch, "The IDEAS project: plug \& produce at shopfloor level," Assembly Automation, 2012.

[17] S. Wrede, O. Beyer, C. Dreyer, M. Wojtynek, and J. J. Steil, "Vertical Integration and Service Orchestration for Modular Production Systems using Business Process Models," 2016.

[18] S. Horbach et al., "Building blocks for adaptable factory systems," in Robotics and Computer-Integrated Manufacturing, 2011.

[19] Jentsch et al., "Towards an Interactive Planning Manual for Production Networks," in XVII Summer School "Francesco Turco", Venice, 2012.

[20] J. Wienke and S. Wrede, "A Middleware for Collaborative Research in Experimental Robotics," in IEEE/SICE International Symposium on System Integration (SII2011). IEEE, 2011, pp. 1183-1190.

[21] Hannover Fair 2017: Robot-based Plug and Produce, (accessed on July 29, 2017), https://www.youtube.com/watch?v=1Lq2YnfV3Xk/. 